\title{
SPECTROSCOPIC VARIABILITY OF THE SUPERGIANTS HD21389 AND HD187982
}

\author{
Y.M.Maharramov, A.Sh.Baloglanov \\ Shamakhy Astrophysical Observatory named after N.Tusi, Azerbaijan National Academy of \\ Sciences, Yu.Mammadaliyev Settlement, Shamakhy district, Azerbaijan \\ y_meherremov@rambler.ru
}

\begin{abstract}
It has been revealed that absorption in the line of $\mathrm{H}_{\alpha}$ has complex structure in the spectrum of the star HD21389 depending on the activity phase of the atmosphere. The profile of the line has inverse P Cyg type in the active phase of the star atmosphere. The emission components in the red and violet wing of the profile form and disappear. All the measured parameters of the $\mathrm{H}_{\alpha}$ and $\mathrm{H}_{\beta}$ lines change. It is supposed that such variations may be due to non-stationary and strong flow substance in the atmosphere of these stars.

Appearance and disappearance of asymmetry in the profile of NaID doublet and formation of the $\mathrm{H}_{\alpha}$ line type inverse P Cyg occurs synchronously in an active phase of the atmosphere of HD 21389. We assume that these changes are formed under the influence of the general mechanism which might be responsible for the observed variation is the growth of the envelope of the star with a following ejection of matter.

Our researches showed that the values of radial velocity of the FeII lines change with time. However, we found no correlation of these changes with the period of 7.7 days found by earlier authors.
\end{abstract}

Key words: stars, radial velocity, spectral parameters, stellar wind.

\section{Introduction}

The supergiant star HD21389 belongs to the stars with P Cyg type profile $\mathrm{H}_{\alpha}$ line. According to (De Jager et al., 1988, Straizys et al., 2007, Takeda et al., 2000, Takeda et al., 1998, Verdugo et al., 1999), its spectral class is A0Ia, apparent magnitude is $\mathrm{m}_{\mathrm{v}}=4^{\mathrm{m}} .53$, mass is $\mathrm{M}_{*} / \mathrm{M}_{\odot}=19.3$, radius is $\mathrm{R}_{*} / \mathrm{R}_{\odot}=97$, luminosity is $\log \left(\mathrm{L}_{*} / \mathrm{L}_{\odot}\right)=4.87$, effective temperature is $\mathrm{T}_{\text {eff }}=10500 \mathrm{~K}$, acceleration of gravity at the surface is $\operatorname{logg}=1.70$, and rotation speed is vsini $=53 \mathrm{~km} / \mathrm{s}$. It is believed that it belongs to the OB1 association.

However, according to other authors (Achmad et al., 1997; De Jager et al., 1988; Talavera et al., 1987; Verdugo et al., 1999), the stellar parameters are somewhat different, $\mathrm{T}_{\text {eff }}=11000 \mathrm{~K}, 9730 \mathrm{~K}$ and $10100 \mathrm{~K}, \mathrm{M}_{*} / \mathrm{M}_{\odot}=14$, $\mathrm{M}_{*} / \mathrm{M}_{\odot}=16, \mathrm{R}_{*} / \mathrm{R}_{\odot}=104.2, \mathrm{R}_{*} / \mathrm{R}_{\odot}=99$.

In (Zeinalov, 1997; Zeinalov, 2003; Zvereva et al., 1984; Rzaev et al., 1989; Takeda et al., 2000; Verdugo et al., 1999; Weaver et al., 1995; Zeinalov et al., 1990), the $\mathrm{H}_{\alpha}, \mathrm{H}_{\beta}, \mathrm{H}_{\gamma}, \mathrm{H}_{\delta}, \mathrm{H}_{\varepsilon}, \mathrm{HeI}(4471,4921,6678 \AA$ ), SiII
(3853.6, 3856, $3862.5 \AA), \mathrm{NaI}$ (D1 and D2), MgII (4481 $\AA)$, DIB $(5797,5780,5850,6614 \AA)$, OI $(6156,6157$, $6158 \AA)$, CII (4267 $\AA)$, FeII (4923.9, 6147.7, $6149.3 \AA)$ lines observed in star spectra were investigated, their equivalent widths were measured, changes were considered, and contours of these lines were presented. It is revealed that the profile of the $\mathrm{H}_{\alpha}$ line has difficult structure and following shapes were observed:

a) normal P Cyg profile;

b) inverse P Cyg profile;

c) pure absorption profile;

e) pure emission profile;

e) $\mathrm{H} \alpha$ exhibiting a three-component shape: the emission profiles on both sides of central absorption component.

One of the interesting moments is that in the profiles of the DIB $\lambda 5797 \AA$ line (Diffuse Interstellar Band) observed in the spectra of this star at different times structural changes are found (Galazutdinov et al., 2004). As strong change happens in the violet wing the equivalent width increases almost twice. Value of equivalent width changes from $\mathrm{W}(5797 \AA)=(74 \pm 4) \mathrm{m} \AA$ to $(156 \pm 7) \mathrm{m} \AA$.

It is revealed that in the spectra of supergiant HD21389, the form, equivalent width, line depth, radial velocity and other parameters of the $\mathrm{H}_{\alpha}$ line show strong change with time. Equivalent width changes from $0.09 \AA$ to $0.45 \AA$, i.e. almost five times (Talavera et al., 1987; Weaver et al., 1995).

In addition, in (Zeinalov, 1997; Zeinalov, 2003; Zvereva et al., 1984; Rzaev et al., 1989; Burki, 1978; Denizman et al., 1988; Gray et al., 1987; Zeinalov et al., 1990) it was noted that the form of the profile of $\mathrm{H}_{\beta}$ line, and also other parameters of this line show strong change. They found even weak emission component in the $\mathrm{H}_{\beta}$ line profiles. On the basis of measurements of the radial velocity on optical depth authors researched the atmosphere of this star (Burki, 1978; Zeinalov et al., 1990). It appeared that the radial velocity determined by the lines $\mathrm{H}_{\alpha}, \mathrm{H}_{\beta}$, HeI and SiII changes on optical depth. They explain these changes with pulsations of the atmosphere of this star.

HD187982 is a supergiant of spectral type A1Iab with the following parameters (Evans et al., 2003; Przybilla et al., 2010; Verdugo et al., 1999):

$$
\begin{aligned}
& \mathrm{m}_{\mathrm{v}}=5^{\mathrm{m}} .58, \mathrm{~T}_{\text {eff }}=(9300 \pm 250) \mathrm{K}, \mathrm{M}_{*} / \mathrm{M}_{\odot}=15, \\
& \mathrm{R}_{*} / \mathrm{R}_{\odot}=78, \operatorname{logg}=1.60 \pm 0.15, \mathrm{vsini}=(15 \pm 6) \mathrm{km} / \mathrm{s} .
\end{aligned}
$$


The supergiant HD187982 belongs to the OB4 associations. In (Abt et al., 1995, Snell et al., 1981, Verdugo et al., 1999) the study of $\mathrm{H}_{\alpha}, \mathrm{H}_{\gamma}, \mathrm{MgII}(4481 \AA$ ), and FeII $(4924,5018,5169 \AA)$ lines observed in the atmospheres of this supergiant is presented. It is noted that generally the profiles of the $\mathrm{H}_{\alpha}$ line are observed in absorption. Sometimes in the red wing of the profile of $\mathrm{H}_{\alpha}$ line is observed weak emission component. A more complete explanation of appearance and disappearance of these components require additional observations.

In the present paper, which can be considered a continuation of the above studies, we analyze variations of the $\mathrm{H}_{\alpha}, \mathrm{H}_{\beta}, \mathrm{HeI}(5876 \AA$ ), NaID and FeII lines in the spectra of HD187982 with the aim to study their peculiarities.

We believe our results will be of interest for further studies of these remarkable stars.

\section{Observation and processing}

Spectral observations of the stars HD21389 and HD187982 in 2005-2014 were carried out using a CCD detector in the echelle spectrometer mounted at the Cassegrain focus of the 2-m telescope of the Shamakhy Astrophysical Observatory (Mikailov et al., 2005). The spectral resolution was $\mathrm{R}=15000$, and the spectral range $\lambda \lambda 4700 \div 6700 \AA$. The signal-to-noise ratio was $\mathrm{S} / \mathrm{N}=200$.

Two spectra of the target stars were obtained on each night of observation. The average exposure was 600-900s, depending on the image quality. Note that, fast changes in the spectra during the night weren't found. Therefore the profiles observed in one night were averaged.

In addition to the observations of the target stars, we also obtained numerous spectra of standard stars, the day and night backgrounds, flat fields, and comparison stars, to enable analysis of the stability of the hardware and telescope-receiver complex.
The Echelle spectra were processed by the standard technique using the DECH20 and DECH20t software (Galazutdinov, 1992).

The errors in our equivalent widths $(\mathrm{W})$ do not exceed $5 \%$, and the errors in the radial velocities $(\mathrm{Vr})$ are no larger than $\sim \pm 2 \mathrm{~km} / \mathrm{s}$. Appropriate heliocentric corrections were included during data processing.

Profiles of the $\mathrm{H}_{\alpha}, \mathrm{H}_{\beta}$, HeI, etc. lines in the spectra of supergiants HD21389 and HD187982 were investigated.

HD21389. The spectra obtained 2005, 2006 and 2014 were investigated. Structures of the profiles and value of radial velocities and spectral parameters of the $H_{\alpha}, H_{\beta}$, HeI and the NaID doublet lines are presented.

The processing of $\mathrm{H}_{\alpha}$ and $\mathrm{H}_{\beta}$ hydrogen lines in the HD21389 spectrum immediately revealed changes in profiles of the $\mathrm{H}_{\alpha}$ line that occur with time. From fig. 1 it is visible that on 20.09.2005 in the spectrum of the supergiant HD21389 the profile of the $\mathrm{H}_{\alpha}$ line is observed in pure absorption and on 11.11.2006 and 11.09.2014 the profile of the line is observed in the inverse and normal $\mathrm{P}$ Cyg profile, respectively (fig. 1).

Unfortunately, as insufficient observation materials we didn't manage to follow these interesting changes in detail.

Apparently from table 1, on 20.09.2005 the radial velocity determined by the $\mathrm{H}_{\alpha}$ and $\mathrm{H}_{\beta}$ lines are equal -29 $\mathrm{km} / \mathrm{s}$ and $-14 \mathrm{~km} / \mathrm{s}$, respectively. However, on 11.11.2006 the radial velocity strongly differs from the values observed on 20.09.2005. In absorption the radial velocity of the $\mathrm{H}_{\alpha}$ lines is $4 \mathrm{~km} / \mathrm{s}$, and in emission is $-69 \mathrm{~km} / \mathrm{s}$. The radial velocities in absorption of the $\mathrm{H}_{\alpha}$ line differ $7-12$ times. Such strong changeability of the radial velocity shows about strong physical process this star occurring in the atmosphere.
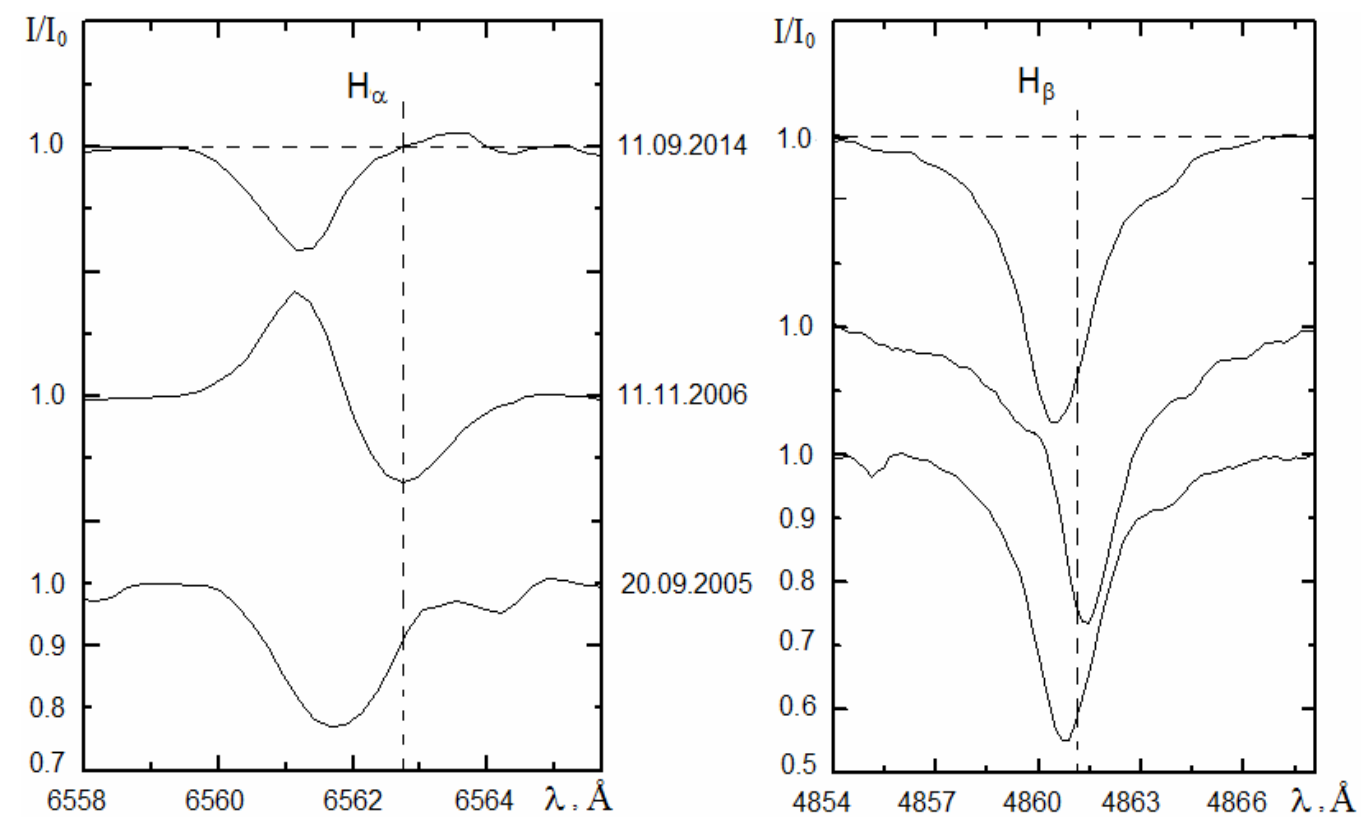

Figure 1: Profiles of the $\mathrm{H}_{\alpha}$ and $\mathrm{H}_{\beta}$ lines of HD21389 on different dates. 


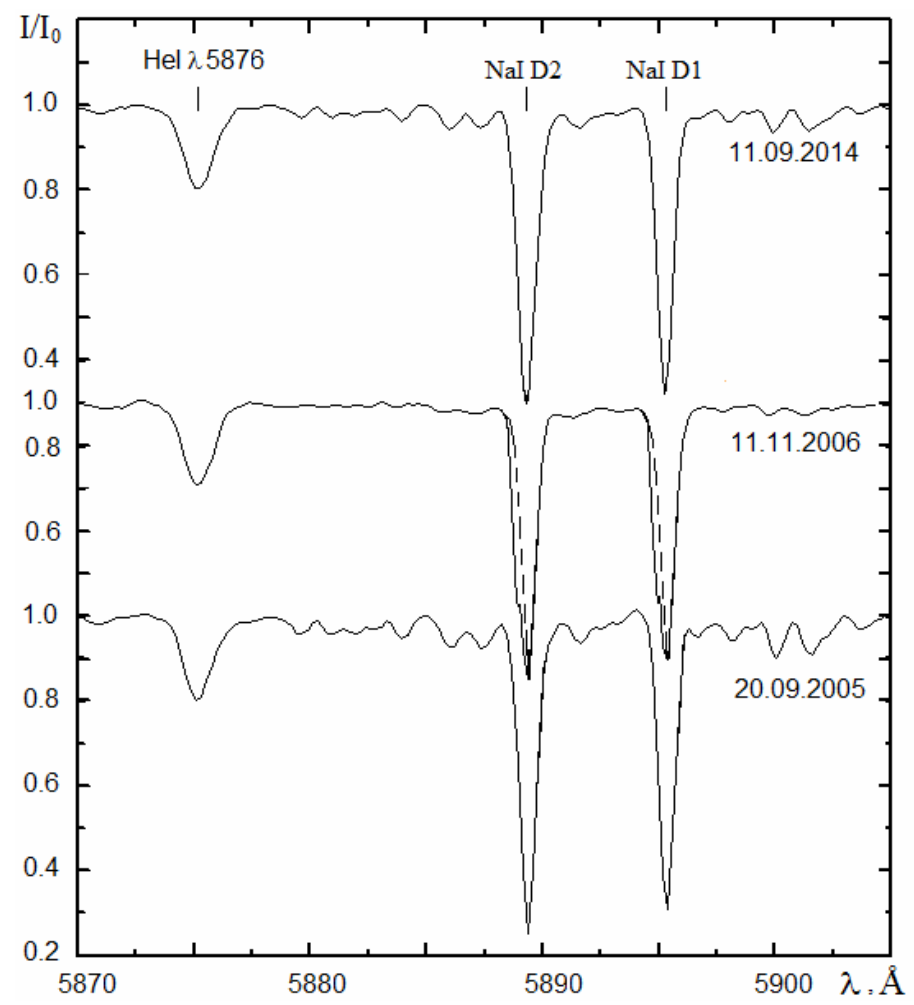

Figure 2: Profiles of the HeI and NaD lines of HD21389 on different dates.

Table 1: Results of measurements of spectral parameters of lines $\mathrm{H}_{\alpha}$, and $\mathrm{H}_{\beta}$

\begin{tabular}{|c|c|c|c|c|c|c|c|c|c|c|c|c|}
\hline \multirow{3}{*}{ Date } & \multicolumn{12}{|c|}{ HD21389 } \\
\hline & \multicolumn{4}{|c|}{$\mathrm{H}_{\mathrm{o}}$ (abs) } & \multicolumn{4}{|c|}{$\mathrm{H}_{3}(\mathrm{em})$} & \multicolumn{4}{|c|}{$\mathrm{H}_{3}$} \\
\hline & $\begin{array}{c}\mathrm{Vr} \\
(\mathrm{km} / \mathrm{s})\end{array}$ & $\tilde{\mathrm{A}}$ & $\begin{array}{c}\Delta \hat{\lambda}_{1 / 2} \\
\AA\end{array}$ & $\mathbf{r}_{v}$ & $\begin{array}{c}\mathrm{Vr} \\
(\mathrm{km} / \mathrm{s})\end{array}$ & W & $\hat{\AA}^{\Delta \hat{\AA}_{1 / 2}}$ & $r_{v}$ & $\begin{array}{c}\mathrm{Vr} \\
(\mathrm{km} / \mathrm{s})\end{array}$ & $\begin{array}{l}\mathrm{W} \\
\hat{\mathrm{A}}\end{array}$ & $\hat{\AA}^{\Delta \hat{\lambda}_{1 / 2}}$ & $\mathbf{r}_{v}$ \\
\hline 20.09 .2005 & -29 & 0.48 & 1.7 & 0.77 & - & - & - & - & -14 & 1.39 & 2.4 & 0.55 \\
\hline 11.11 .2006 & 4 & 0.19 & 1.3 & 0.86 & -69 & 0.19 & 1.1 & 1.17 & 10 & 1.68 & 2.2 & 0.53 \\
\hline 11.09 .2014 & -49 & 0.39 & 1.5 & 0.81 & - & - & - & - & -30 & 1.55 & 2.7 & 0.55 \\
\hline \multirow{3}{*}{ Date } & \multicolumn{12}{|c|}{ HD187982 } \\
\hline & \multicolumn{4}{|c|}{$\mathrm{H}_{\mathrm{\alpha}}$ (abs) } & \multicolumn{4}{|c|}{$\mathrm{H}_{\mathrm{a}}(\mathrm{em})$} & \multicolumn{4}{|c|}{$\mathrm{H}_{3}$} \\
\hline & $\begin{array}{c}\mathrm{Vr} \\
(\mathrm{km} / \mathrm{s})\end{array}$ & $\begin{array}{l}\mathrm{W} \\
\AA\end{array}$ & $\hat{\AA}_{1 / 2}$ & $\mathbf{r}_{v}$ & $\begin{array}{c}\mathrm{Vr} \\
(\mathrm{km} / \mathrm{s})\end{array}$ & $\begin{array}{l}\mathrm{W} \\
\hat{\mathrm{A}}\end{array}$ & $\hat{\mathrm{A}}^{\Delta \hat{\lambda}_{1 / 2}}$ & $\mathrm{I}_{v}$ & $\begin{array}{c}\mathrm{Vr} \\
(\mathrm{km} / \mathrm{s})\end{array}$ & $\begin{array}{l}\mathrm{W} \\
\AA \\
\AA\end{array}$ & $\overline{\mathrm{A}}_{1 / 2}^{\mathrm{A}}$ & $\mathbf{r}_{v}$ \\
\hline 01.09 .2013 & -29 & 0.89 & 2.5 & 0.72 & 79 & 0.02 & 0.58 & 1.04 & -33 & 2.46 & 3.1 & 0.47 \\
\hline 06.09 .2013 & -23 & 0.77 & 2.6 & 0.76 & 70 & 0.03 & 0.72 & 1.04 & -33 & 2.26 & 3.2 & 0.48 \\
\hline 02.10 .2013 & -18 & 1.29 & 2.9 & 0.69 & - & - & - & - & -18 & 2.40 & 3.0 & 0.43 \\
\hline 03.10 .2013 & -17 & 1.33 & 2.6 & 0.68 & - & - & - & - & -19 & 2.42 & 3.2 & 0.45 \\
\hline 21.06 .2014 & -11 & 1.06 & 2.3 & 0.67 & - & - & - & - & -16 & 2.35 & 2.9 & 0.42 \\
\hline 04.07 .2014 & -5 & 1.15 & 2.3 & 0.63 & - & - & - & - & -9 & 2.53 & 2.7 & 0.39 \\
\hline 11.07.2014 & -6 & 1.10 & 2.1 & 0.63 & - & - & - & - & -13 & 2.41 & 2.7 & 0.40 \\
\hline 18.07 .2014 & -5 & 1.33 & 2.5 & 0.62 & - & - & - & - & -8 & 2.57 & 3.0 & 0.38 \\
\hline 24.07 .2014 & -12 & 1.36 & 2.4 & 0.59 & - & - & - & - & -14 & 2.41 & 2.7 & 0.37 \\
\hline 09.08 .2014 & -13 & 1.38 & 2.6 & 0.59 & - & - & - & - & -12 & 2.80 & 3.2 & 0.39 \\
\hline
\end{tabular}

As shown on 11.11.2006, we observed an inversion of the P Cyg profile of the $\mathrm{H}_{\alpha}$ line. The inverse P-Cygprofile may be explained by the high-velocity motion of wind matter away from the observer. This suggests that, as a result of a strong stellar wind, the absorption component of the $\mathrm{H}_{\alpha}$ line is shifted to the red region of the profile while the emission component is blueshifted.

From fig. 1 and tab. 1 it is visible that when the $H_{\alpha}$ is observed inverse P Cyg, the structure of the profile of $\mathrm{H}_{\beta}$ changed a little and this line displaced to longer wavelength. It is interesting that when the $\mathrm{H}_{\alpha}$ is observed inverse P Cyg, the profile of HeI (5876 A) line does not change. It means that in this case, the deep layers of the HD21389 atmosphere are relatively stable.

In addition, from tab. 1 it is visible that during an inversion event, in the $H_{\alpha}$ line half-width $\left(\Delta \lambda_{1 / 2}\right)$ and equivalent width decrease, and residual intensity grows. At the same time, in the $\mathrm{H}_{\beta}$ half-width decreased and the 
residual intensity changed very little, at the same time equivalent width on the contrary increased. And from tab. 2 it is visible that in HeI the residual intensity, half-width and equivalent width didn't change.

The spectrum observed on 11.09.2014 shows that in the red wing of the $\mathrm{H}_{\alpha}$ line are present weak emission component (fig. 1). When comparing it became clear that in this case, the $\mathrm{H}_{\alpha}$ and $\mathrm{H}_{\beta}$ lines were strongly displaced to the violet side of the spectrum and radial velocity became more than 11.11.2006 (fig. 1 and tab. 1). This time in the HeI lines $(5876 \AA)$ and NaID doublet any change hasn't occurred.

Unfortunately, as insufficient observation material we can't reveal certain regularity.

HD187982. Profile of the $\mathrm{H}_{\alpha}$ line is P Cyg type. On the basis of the observed spectra the profiles of the $\mathrm{H}_{\alpha}, \mathrm{H}_{\beta}$, HeI (5876 A), NaID doublet and FeII (4924, 5018, 5169 $\AA)$ lines were investigated. The radial velocities, residual intensities and half-widths of the studied lines are determined. In the spectra of HD187982 observed on 01.09 and on 06.09.2014 the profiles of the $H_{\alpha}$ lines consist of a strong absorption component and a weak emission component which is observed on the red wing of the $\mathrm{H}_{\alpha}$ line (fig. 3). It is also interesting that from emission component of the $\mathrm{H}_{\alpha}$ line to longer wavelength there is a weak absorption component again. But in the spectra of 02.10.2013 and 03.10.2013 the $\mathrm{H}_{\alpha}$ line is visible only in absorption and there are no accompanying components.

Apparently from fig. 3 and 4 , in all cases, in the profiles of the $\mathrm{H}_{\beta}$ and $\mathrm{NaI}$ lines structural changes aren't observed. If we follow the radial velocities of $\mathrm{H}_{\alpha}, \mathrm{H}_{\beta}, \mathrm{HeI}$ and NaID doublet lines, we will see that the radial velocity of $\mathrm{H}_{\alpha}$ line changes, but no significant variability was observed. In the HeI lines and the NaID doublet the changing of $\mathrm{Vr}$ is very weak.

It was revealed that change of the radial velocity in the $\mathrm{H}_{\beta}$ line shows interesting similarity to the form of $\mathrm{H}_{\alpha}$ profile. As it was underlined above, in the spectra of HD187982 star the profile of the $\mathrm{H}_{\alpha}$ line is observed in two following forms:

a) the profile of the $\mathrm{H}_{\alpha}$ line consists of a strong absorption component and a weak emission component which is observed on the red wing of the $\mathrm{H}_{\alpha}$ line.

b) the profile of the $\mathrm{H}_{\alpha}$ line is observed in pure absorption.

On $\quad 01.09 .2013, \quad 06.09 .2013,02.10 .2013$ and 03.10.2013 dates in the spectra of HD187982 star the radial velocity of the $\mathrm{H}_{\beta}$ line there were $-33 \mathrm{~km} / \mathrm{s},-33$ $\mathrm{km} / \mathrm{s},-18 \mathrm{~km} / \mathrm{s}$ and $-19 \mathrm{~km} / \mathrm{s}$, respectively.

Apparently, upon transition of the $\mathrm{H}_{\alpha}$ profile from a form a) to the b) case the $\mathrm{H}_{\beta}$ line moves to the red side, and the radial velocity changes sharply, but in spectral parameters no significant variability was observed (tab. 1).

In addition, upon such transition the equivalent width of the $\mathrm{H}_{\alpha}$ increases, but in half-width and in residual intensity no significant variability was observed. At the same time in the radial velocity and spectral parameters of the HeI and NaID doublet lines noticeable changes weren't observed. The further spectra of this star are observed between 21.06.2014 and 09.08.2014. In the spectra observed on 21.06 09.08.2014 dates the radial velocity of $\mathrm{H}_{\alpha}$ and $\mathrm{H}_{\beta}$ changed on average on $\pm 4 \mathrm{~km} / \mathrm{s}$. But in the structure of profiles of these lines, and also in values of the radial velocities of HeI and NaID doublet lines, strong changes aren't revealed.

Table 2: Results of measurements of spectral parameters of lines HeI, and NaID

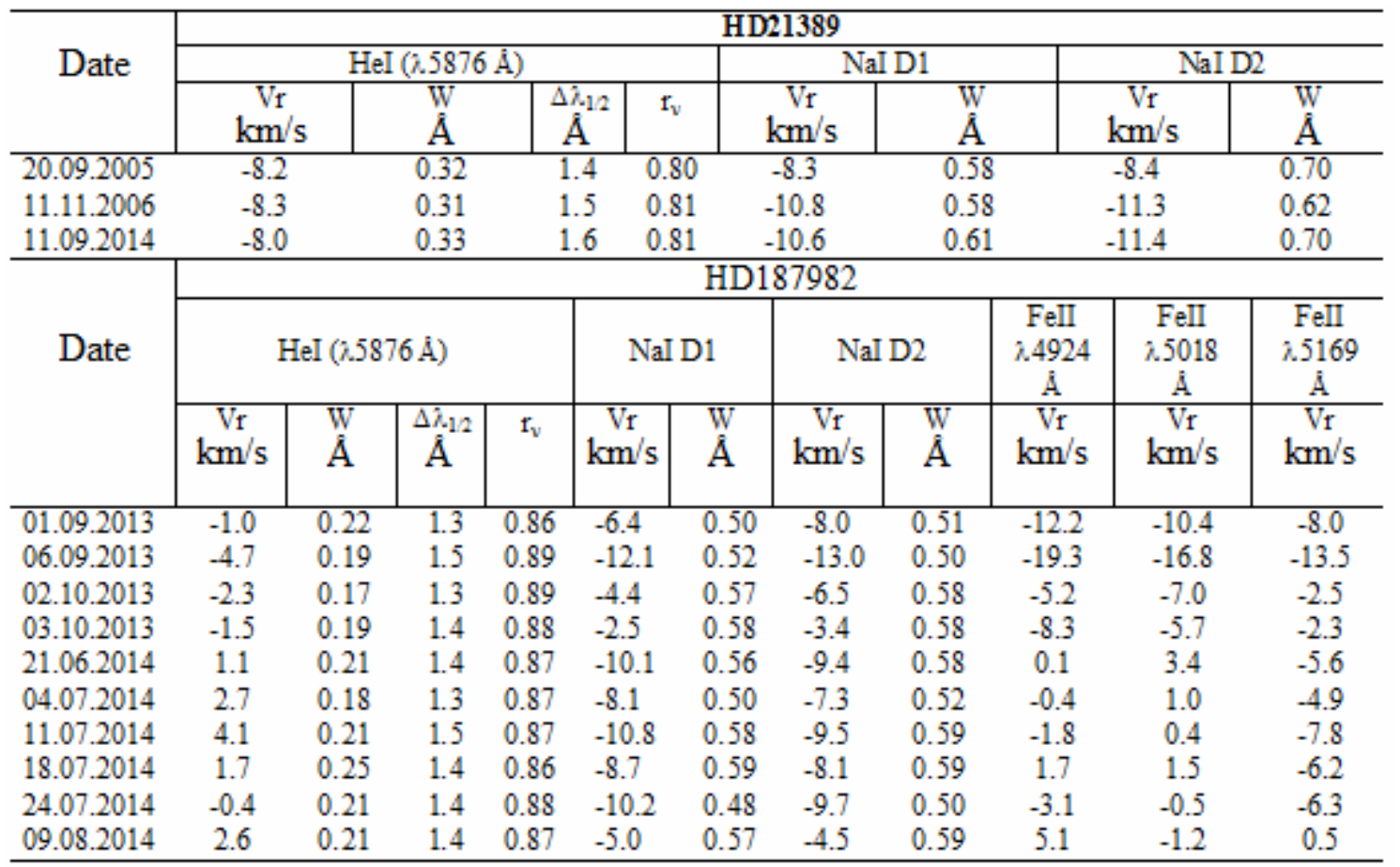



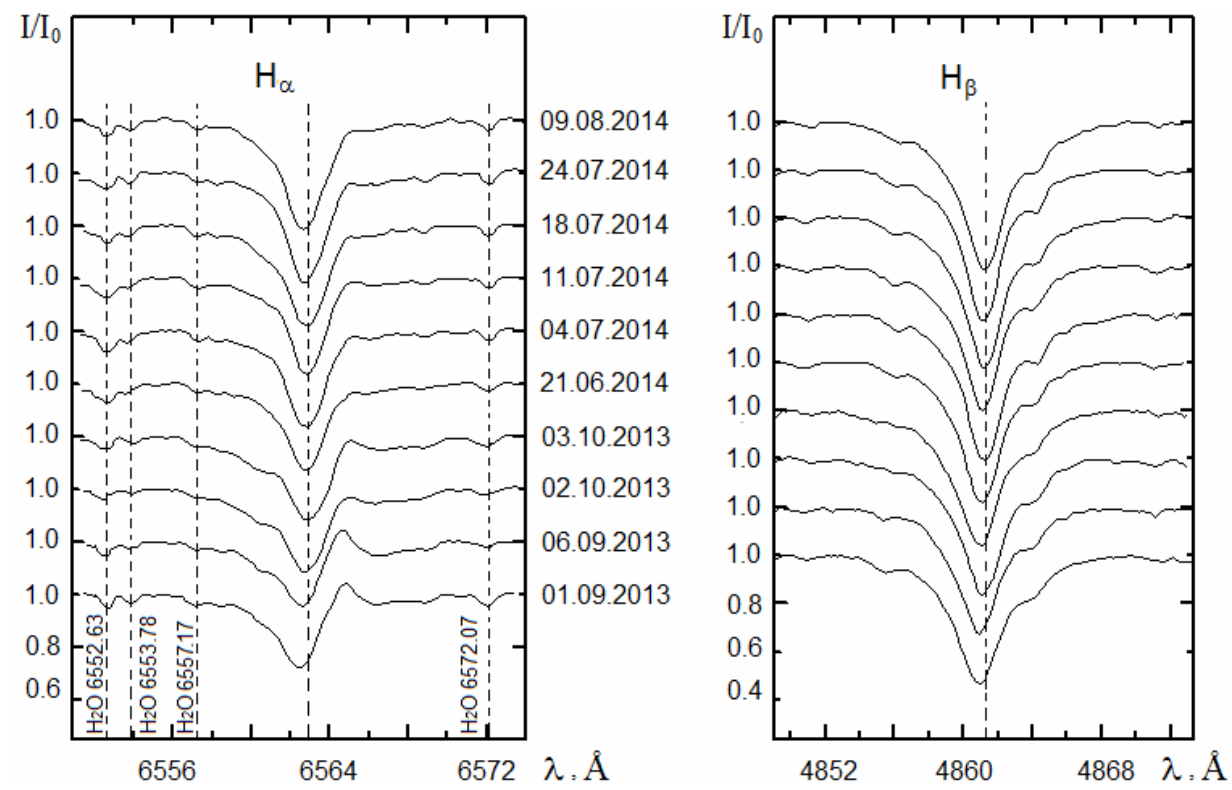

Figure 3: $H_{\alpha}$ and $H_{\beta}$ line profiles of the star HD187982 in 2013-2014.

On the other hand, changes of the radial velocity of the FeII line were also considered. Note that the FeII lines in the spectra of HD187982 were detailed investigated by White (1950), Abt (1957) and Hendry (1981). We tried to continue the works of these researchers and to compare the results. In Abt's (1957) work for the radial velocity it is found 7.7 days quasiperiodic change on the basis of the FeII lines (4508, 4515, 4520, $4522 \AA$ ). After Abt (1957) the measurements were carried out by Hendry (1981). He added to measurements of White (1950) and Abt's data and received same earlier found 7.7 days period.

On the basis of the spectrograms obtained by us, and also observational data of other authors taken from works White (1950), Abt (1957) and Hendry (1981) were constructed graphics of change of the radial velocity in the FeII lines. Our researches showed that the radial velocities of the FeII lines change with time. However, we didn't find periodicity in these changes. To reveal periodic processes additional observation materials is necessary.

NaI $5890 \AA$ and $5896 \AA$. In the spectra of the stars HD 21389 and HD187982, except hydrogen and other lines,

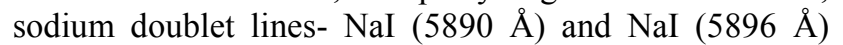
were also investigated.

It is known that generally the absorption of $\mathrm{NaI}(5890$ $\AA$ and $5896 \AA$ ) lines are interstellar lines, i.e. are formed in the interstellar environment. Therefore the profiles of these lines have to be symmetric. Asymmetry of these profiles is argument in favor of the contribution of other sources to formation of these lines.

Investigation on optical spectra of these stars, and also other hot supergiants observed by us and other authors, specify that in formation of NaID doublet, except a star covers round these stars and the interstellar environment can make a contribution.

Unfortunately, as small resolution in the spectra, we couldn't reveal these components. However, on 11.11.2006 in the spectrum of HD 21389 in the profile of NaID doublet is observed asymmetry in violet wings of these lines. Therefore, for detection of asymmetry, we combined the red wing of these lines with a Gaussian profile. In fig. 2 the dashed line showed combination of the red wing with a Gaussian profile.
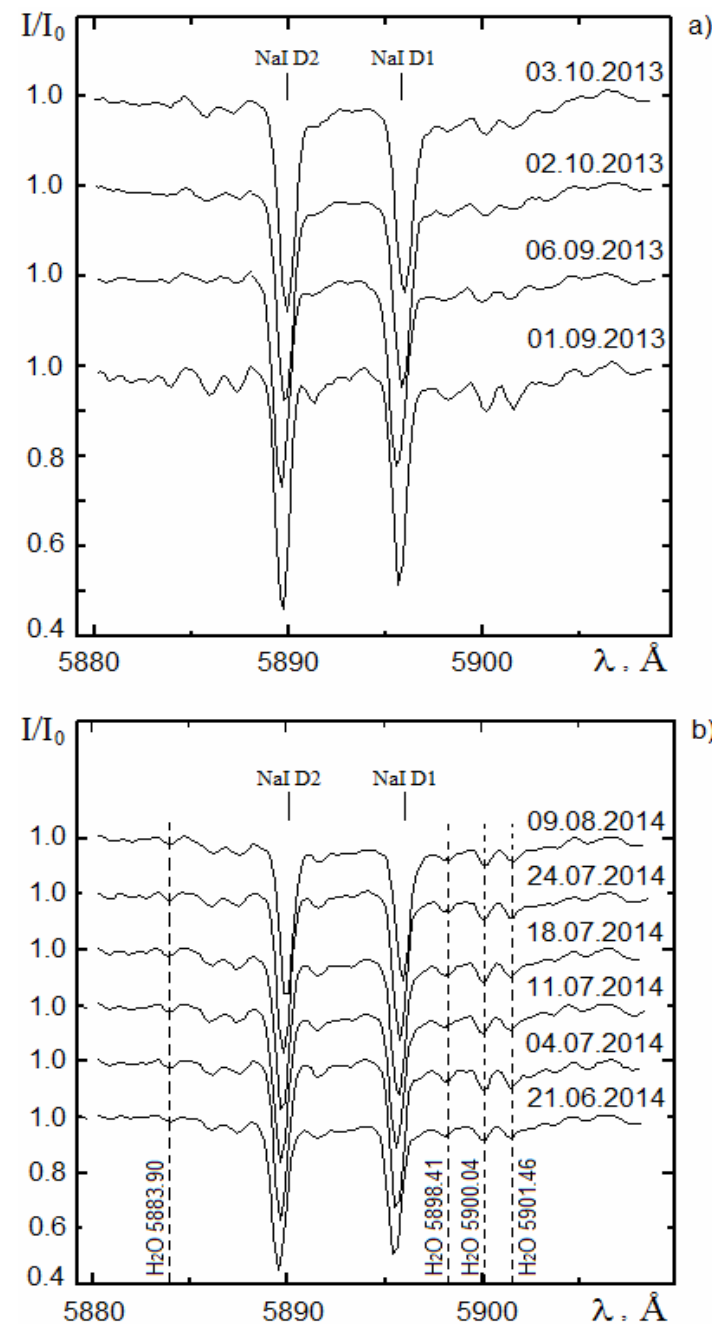

Figure 4: $\mathrm{NaD}$ line profiles of the star HD187982 in 2013-2014. 
The radial velocities of studied lines are determined by combination of straight lines and mirror images of the specified lines of absorption in the level of the half-width $\mathrm{Vr}(\mathrm{R} / 2)$, and also $\mathrm{Vr}$ in the centers of the profiles. Laboratory lengths of waves (D2, D1) were accepted as $5889.953 \AA$ and $5895.923 \AA$, respectively. The values of the radial velocities and equivalent width $\left(\mathrm{W}_{\lambda}\right)$ determined by us are given in table 2 .

Apparently from table 2, in the spectra of HD187982 supergiant the equivalent width of the $\mathrm{NaI}(5890 \AA)$ and NaI (5896 $\AA$ ) lines determined by us significantly changes aren't observed. However, the radial velocities of these lines variability are observed.

In addition, only on 11.11.2006 in the spectra of the star HD 21389 the asymmetry is observed in the NaID doublet line. Appearance and disappearance of asymmetry in the profile of NaID doublet and formation of the $\mathrm{H}_{\alpha}$ line type inverse $\mathrm{P}$ Cyg happens synchronously in an active phase of the atmosphere of HD 21389 (fig. 1, 2).

\section{Discussion}

The profile of the $\mathrm{H}_{\alpha}$ line observed in the atmosphere of the HD21389 and HD187982 supergiants indicates variable structure. The analysis of the absorption and emission components of the $\mathrm{H}_{\alpha}$ line shows that the radial velocities change with time. Our investigation shows that all the measured parameters of $\mathrm{H}_{\beta}$ line and its radial velocities vary with time.

It is known that the $\mathrm{H}_{\alpha}$ and $\mathrm{H}_{\beta}$ lines form in the upper layers of the stellar atmosphere, in the region of generation of stellar wind (De Jager, 1984). The variable wind and its accelerated motion in supergiants is caused by the strong flux of radiation from the star. Outer atmospheres of supergiant stars are exposed to more intense changes than internal.

Thus, the stellar radiation flux and the variable stellar wind lead to corresponding changes in the outer layers of the atmosphere and the star envelope.

As a result, we observe variable absorption and emission components of different forms of the $\mathrm{H}_{\alpha}$ line $\mathrm{P}$ Cyg-profile of the star HD21389 and HD187982.

As is known, the variable stellar wind in the supergiants is caused by the pulsation (Cox, 1983). If these changes in the stars HD21389 and HD187982 are associated with the pulsation, they should occur periodically. The amount of obtained data and their inconsistency in observation time does not make it possible to make such far-reaching conclusions in this paper.

Therefore, additional systematic spectral and photometric observations of these stars are planned at the Shamakhy Astrophysical Observatory in the near future.

In addition, as it is noted above, asymmetry of interstellar lines of $\mathrm{NaI} 5890$ (D2) and $\mathrm{NaI} 5896$ (D1) in the spectra of the supergiant HD 21389 (fig.2) is found. In all the specified lines an echelle spectrograms asymmetry observed by us it is found only in violet wings of these lines and only 11.11.2006, when absorption profiles the $\mathrm{H}_{\alpha}$ line is observed in the form of inverse $\mathrm{P}$ Cyg. However, in 20.09.2005 and 11.09.2014 the asymmetry in the profile of a NaID doublet isn't observed. From fig. 1 it is visible that in 20.09.2005 in the spectra of HD21389 supergiant the profile of the $\mathrm{H}_{\alpha}$ line is observed in pure absorption, and in 11.09.2014 - normal P Cyg profile when on the red wing of $\mathrm{H}_{\alpha}$ line very weak emission component is observed (fig. 1).

But the asymmetry in the profiles of NaID doublet isn't found by us in the spectra of other supergiant HD187982. This observational fact is additional argument in favor of reality of the asymmetry of the studied NaI 5890 (D2) and NaI 5896 (D1) lines revealed by us in the spectra of the star HD 21389.

We assume that the asymmetry of the specified absorption lines in the spectra of supergiant HD21389 found by us and change of the profile of $\mathrm{H}_{\alpha}$ line are formed under the influence of the general mechanism which might be responsible for the observed variation is the growth of the envelope of the star with a following ejection of matter.

\section{Conclusions}

1. The absorption profile of the $\mathrm{H}_{\alpha}$ line in the spectra of the star HD21389 depending on a phase of instability of the star atmosphere has difficult structure. In an active phase this line has inverse P Cyg profile. On the red and violet wings of the profile appear and disappear emission components. All measured parameters of the $\mathrm{H}_{\alpha}$ line show variability. It is supposed that variability of the profiles, radial velocity and other parameters are connected with each other and also these changes may be a manifestation of complex motions in the atmosphere of HD21389.

2. Appearance and disappearance of asymmetry in the profile of NaID doublet and formation of the $\mathrm{H}_{\alpha}$ line type inverse P Cyg occurs synchronously in an active phase of the atmosphere of HD 21389. We assume that these changes are formed under the influence of the general mechanism which might be responsible for the observed variation is the growth of the envelope of the star with a following ejection of matter.

3. Our researches showed that the values of radial velocity of the FeII lines change with time. However, we found no correlation of these changes with the period of 7.7 days found by earlier authors (Abt, 1957, Hendry, 1981).

These results can be used for the further analysis of a chemical composition and determination of fundamental parameters of the atmosphere, and also for creation of the theoretical model of this star.

Acknowledgments. This work was supported by the scientific program for the priority fields of research of the National Academy of Sciences of Azerbaijan.

\section{References}

Achmad L., Lamers H.J.G.L.M., L.Pasquini L.: 1997, Astronomy \& Astrophysics, 320, 196.

Burki G.: 1978, Astronomy \& Astrophysics, 65, 357.

Cox J.P.: 1983, Theory of Stellar Pulsation, Mir, Moscow. De Jager C.: 1984, The Brightest Stars, Mir, Moscow.

De Jager C., Nieuwenhuijzen H., van der Hucht K.A.: 1988, Astronomy \& Astrophysics, Suppl. Ser., 72, 259. 
Denizman L., Hack M.: 1988, Astronomy \& Astrophysics, 75, 79.

Evans C.J., Howarth Ian D.: 2003, MNRAS, 345, 1223.

Galazutdinov G.A.: 1992, Prepr. SAO RAS, 92, 2.

Galazutdinov G.A., Manico G., Pirronello V., Krelowski J.: 2004, MNRAS, 355, 169.

Gray R.O., Garisson R.F.: 1987, Astrophysical Journal, Suppl. Ser., 65, 581.

Helmut A.A., Nidia I.Morrell.: 1995, Astrophysical Journal, Suppl. Ser., 99, 135.

Helmut A.A.: 1957, Astrophysical Journal, 126, 138.

Hendry E.M.: 1981, The Astronomical Journal, 86, 1540.

Mikailov Kh.M., Khalilov V.M., Alekberov I.A.: 2005, Tsirk. ShAO, 109, 21.

Przybilla N., Firnstein M., Nieva M.F., Meynet G., Maeder A.: 2010, Astronomy \& Astrophysics, 517, 1.

Rzaev A.Kh., Zeinalov S.K., Chentsov E.L.: 1989, Kinematics and Physics of Selectial Bodies, 5, 81.

Snell R.L., Vanden Bout P.A.: 1981, Astrophysical Journal, 244, 844.

Straizys V., Laugalys V.: 2007, Baltic Atronomy, 16, 167.

Takeda Y., Takada-Hidai M.: 2000, Japan Astronomical Society, 52, 113.
Takeda Y., Takada-Hidai M.: 1998, Japan Astronomical Society, 50, 629.

Talavera A., de Castro A.I.Gomez.: 1987, Astronomy \& Astrophysics, 181, 300.

Verdugo E., Talavera A., de Castro A.I.Gomez.: 1999, Astronomy \& Astrophysics, 346, 819.

Verdugo E., Talavera A., de Castro A.I.Gomez.: 1999, Astronomy \& Astrophysics, 137, 351.

Weaver Wm. B., Torres-Dodgen Ana V.: 1995, Astrophysical Journal, 446, 300.

White W.C.: 1950, Astrophysical Journal, 112, 559.

Zeinalov S.K., Rzaev A.Kh.: 1990, Astrophysics and Space Science, 172, 211.

Zeinalov S.K., Rzaev A.Kh.: 1990, Astrophysics and Space Science, 172, 217.

Zeinalov S.K.: 1997, Doctoral dissertation, The nonstationary phenomena in the atmosphere of $O, B, A, F$ supergiants, Tbilisi.

Zeinalov S.K.: 2003, Tsirk. ShAO, 106, 22.

Zvereva E.B., Zeinalov S.K., Chentsov E.L.: 1984, Izv. Spets. Astrophys. Obs. 18, 29. 